\title{
CRESCIMENTO E DESENVOLVIMENTO DO ALGODOEIRO EM DIFERENTES CONFIGURAÇÕES DE SEMEADURA ( $\left.{ }^{1}\right)$
}

\author{
ARIANA VIEIRA SILVA $\left({ }^{2}\right)$; EDERALDO JOSÉ CHIAVEGATO $\left({ }^{3 *}\right)$; LUIZ HENRIQUE CARVALHO $\left({ }^{4}\right)$; \\ DANIELA MOREIRA KUBIAK $\left({ }^{5}\right)$
}

\begin{abstract}
RESUMO
Foram avaliados o crescimento e o desenvolvimento da cultivar de algodoeiro IAC 23 em espaçamentos ultra-adensado, adensado e convencional entre fileiras e com número variado de plantas por metro linear. O experimento foi implantado na área experimental da Universidade de São Paulo/ Escola Superior de Agricultura “Luiz de Queiroz", Piracicaba (SP), no ano agrícola 2001/2002. O delineamento experimental compreendeu um esquema fatorial, em blocos ao acaso, com 4 repetições, sendo três espaçamentos entre linhas $(0,38 ; 0,76$ e $0,95 \mathrm{~m})$ e quatro densidades de plantas por metro linear $(5,8,11$ e 14). Os parâmetros avaliados foram: índice de área foliar (IAF) e massa seca da parte aérea em todos os estádios da cultura (aparecimento do primeiro botão floral; abertura da primeira flor; maturação fisiológica; primeiro capulho; completa maturação); altura média de plantas, altura de inserção do $1 .^{\circ}$ ramo frutífero, diâmetro do caule, número de ramos vegetativos e frutíferos e número de internódios, verificados por ocasião da colheita. Nas condições estudadas, a cultivar completou seu ciclo em 161 dias. Constatou-se interação significativa entre espaçamento e densidade para altura média das plantas, número de ramos frutíferos e de internódios e que diferentes configurações de semeadura alteram o crescimento e o desenvolvimento do algodoeiro.
\end{abstract}

Palavras-chave: algodoeiro, espaçamento, densidade, índice de área foliar, massa seca, altura de plantas, altura de inserção do primeiro ramo frutífero, diâmetro de caule.

\section{ABSTRACT \\ GROWTH AND DEVELOPMENT OF COTTON PLANT UNDER DIFFERENT SOWING CONFIGURATIONS}

The growth and development of cotton IAC 23 under ultra-narrow, narrow and conventional rows and different numbers of plants for linear meter have been evaluated. The experiment was lead in the experimental area of the University of São Paulo/ESALQ, Piracicaba, State of São Paulo, Brasil, in 2001/ 2002. The experimental design was a randomized complete block, in factorial scheme, with 4 replications, being three rows $(0.38 ; 0.76$ and $0.95 \mathrm{~m})$ and four densities of plants for linear meter $(5,8,11$ and 14$)$. They were analyzed: leaf area index (LAI) and plant shoot dry mass in all phenological phases (emergence of first flower buds, opening of first flower; physiological maturation; first open boll; complete maturation); average plant height, insertion of the first fruit branch, stem diameter, number of vegetative

(1) Parte do trabalho de dissertação do mestrado pela Universidade de São Paulo/Escola Superior de Agricultura “Luiz de Queiroz". Trabalho apresentado no III Congresso Brasileiro de Algodão, Goiânia (GO), 2003. Recebido para publicação em 31 de agosto de 2004 e aceito em 12 de maio de 2006.

$\left(^{2}\right)$ Doutoranda do Programa de Pós-Graduação em Fitotecnia, Escola Superior de Agricultura "Luiz de Queiroz", Universidade de São Paulo, Caixa Postal 9, 13418-900 Piracicaba (SP). E-mail: avsilva@esalq.usp.br. Bolsista CNPq.

$\left({ }^{3}\right)$ Departamento de Produção Vegetal, Escola Superior de Agricultura “Luiz de Queiroz”, Universidade de São Paulo. E-mail: ejchiave@esalq.usp.br. *Autor correspondente.

$\left({ }^{4}\right)$ Instituto Agronômico (IAC) Caixa Postal 28, 13001-970, Campinas (SP). E-mail: 1hcarval@iac.sp.gov.br

$\left(^{5}\right)$ Doutoranda do Programa de Pós-Graduação em Fitotecnia, Escola Superior de Agricultura "Luiz de Queiroz", Universidade de São Paulo. E-mail: dmkubiak@esalq.usp.br. Bolsista CAPES. 
and fruit branches and number of internodes, were estimated by harvest occasion. Under the studied conditions, the cultivar completed its cycle in 161 days. Interaction between rows and densities of plants occurred to average plant height; number of fruit branches and internodes. Different configurations of rows modified the growth and the development of the cotton plants.

Key words: cotton, row, density, leaf area index, dry mass, height of plants, insertion of the first fruit branch, stem diameter.

\section{INTRODUÇÃO}

O crescimento e o desenvolvimento do algodoeiro são dependentes da configuração de semeadura, a qual ocasiona mudanças nas características morfológicas e fisiológicas da planta e da cultura como um todo (FowLER e RAY, 1977).

Dentre os espaçamentos possíveis, verificamse o ultra-adensado, utilizado em algumas regiões dos EUA, compreendendo o dimensionamento entre linhas de 0,19 a 0,38 m (Jost e Cothren, 1999); o adensado, de 0,39 a $0,76 \mathrm{~m}$ (WeIr, 1996) e o convencional, com espaçamentos superiores a $0,76 \mathrm{~m}$.

ReEves (2000) ressalta que a melhor conservação do solo pode ser obtida com a adoção do espaçamento ultra-adensado, além de proporcionar maior rentabilidade aos produtores. Nos tradicionais Estados cotonicultores brasileiros como São Paulo, Paraná, Minas Gerais e em algumas áreas nos cerrados do Centro-Oeste, com os solos já há muito explorados, erodidos, compactados, com altos teores de alumínio e baixos teores de matéria orgânica, a adoção de espaçamentos ultra-adensados pode ser vantajosa se comparada aos espaçamentos convencionais. Segundo REEves (2000), aliado a um sistema conservacionista, essa configuração de semeadura diminui os custos com insumos agrícolas, aumenta a rentabilidade em curto prazo, além de conservar e/ou melhorar as características do solo e a produtividade em longo prazo. O cultivo do algodoeiro em altas densidades populacionais vem recebendo especial atenção de produtores e pesquisadores, sobretudo nos Estados Unidos.

Assim, o estudo teve por objetivo avaliar diferentes configurações de semeadura, comparandose os espaçamentos ultra-adensado, adensado e convencional em diferentes densidades populacionais e suas implicações no crescimento e desenvolvimento das plantas do algodoeiro, com vistas no manejo dessa cultura em nosso meio.

\section{MATERIAL E MÉTODOS}

O experimento foi desenvolvido no ano agrícola de 2001/2002, na área experimental da USP /
ESALQ, Piracicaba (SP), em solo classificado como Terra Roxa Estruturada Eutrófica. O clima da região é do tipo Cwa (clima mesotérmico, úmido, subtropical com inverno seco), segundo a classificação de Koeppen (Vianello e Alves, 1991).

A semeadura foi realizada em 10 de novembro de 2001. O delineamento experimental compreendeu um esquema fatorial, com 3 espaçamentos entre linhas $(0,38 ; 0,76$ e $0,95 \mathrm{~m})$ e 4 densidades $(5,8,11$ e 14 plantas por metro linear), distribuídos em blocos ao acaso, com 4 repetições. Foi utilizada a cultivar IAC 23, por ser resistente às principais doenças e nematóides, empregando-se sementes tratadas com carbofuran e carboxin.

As adubações de semeadura e de cobertura foram realizadas por unidade de área, independentemente do espaçamento utilizado; no sulco de semeadura foram utilizados $375 \mathrm{~kg}$.ha- ${ }^{-1}$ da fórmula 4-20-20 e, em cobertura, aos 30 dias após a emergência, foram empregados $90 \mathrm{~kg} \cdot \mathrm{ha}^{-1}$ de nitrogênio, na forma de sulfato de amônia. Para adequar o crescimento das plantas aos sistemas de produção estudados, foi utilizado o regulador vegetal cloreto de mepiquat (PIX), de tal forma que as plantas no espaçamento de 0,$38 ; 0,76$ e $0,95 \mathrm{~m}$ atingissem as alturas finais de 0,$60 ; 1,15$ e $1,40 \mathrm{~m}$ conforme Righi et al. (1965); Passos (1977); GridiPApp et al. (1992). O manejo das plantas daninhas e fitossanitário foram realizados conforme a necessidade.

As variáveis índice de área foliar (IAF) e massa seca da parte aérea (g) foram avaliadas em todos os estádios da cultura (aparecimento do primeiro botão floral; abertura da primeira flor; maturação fisiológica; primeiro capulho; completa maturação); a altura média de plantas (m), altura de inserção do $1 .^{\circ}$ ramo frutífero $(\mathrm{cm})$, diâmetro do caule $(\mathrm{cm})$, número de ramos vegetativos e frutíferos e número de internódios, foram verificados por ocasião da colheita, manual e realizada aos 151 dias após a emergência. Os dados coletados foram submetidos à análise de variância e as médias de tratamentos comparadas pelo teste de Tukey a $5 \%$ de probabilidade. 


\section{RESULTADOS E DISCUSSÃO}

Nas condições estudadas, observaram-se na cultivar crescimento e desenvolvimento normais na cultivar, completando o ciclo em 161 dias da semeadura a última colheita.

Nos estádios fenológicos considerados, o índice de área foliar (IAF) foi influenciado estatisticamente pelo espaçamento e pela densidade quando estudados isoladamente, sem efeito dessa interação. No estádio de $1 .^{\circ}$ capulho, o índice de área foliar no espaçamento de $0,38 \mathrm{~m}$ foi estatisticamente superior ao do espaçamento de $0,95 \mathrm{~m}$, e na densidade de 14 plantas $\mathrm{m}^{-1}$ linear foi significativamente maior que das demais densidades.

A massa seca da parte aérea foi influenciada pelo espaçamento em todos os estádios fenológicos estudados, e pela densidade nos estádios de $1 .^{\mathrm{a}}$ flor branca e maturação fisiológica, não ocorrendo interação significativa entre o espaçamento e a densidade. Em todos os estádios, a massa seca da parte aérea foi significativamente inferior no espaçamento de $0,38 \mathrm{~m}$ quando comparado aos espaçamentos de 0,76 e $0,95 \mathrm{~m}$, os quais não diferiram entre si estatisticamente.

Para a altura média das plantas, por ocasião da colheita, houve efeito significativo de espaçamento, densidade e da interação entre eles. Nas três menores densidades, no espaçamento de $0,38 \mathrm{~m}$, a altura foi estatisticamente mais baixa quando comparada ao espaçamento convencional $(0,76 \mathrm{~m})$, não diferindo na maior densidade. No espaçamento de $0,38 \mathrm{~m}$ e nas densidades de 8 e 11 plantas. $\mathrm{m}^{-1}$ linear, as plantas cresceram menos, significativamente, do que no espaçamento de $0,76 \mathrm{~m}$ com as mesmas densidades (Tabela 1).

Para a inserção do $1 .^{\circ}$ ramo frutífero, avaliada por ocasião da colheita, não houve interação significativa entre espaçamento e densidade, mas observou-se efeito das densidades estudadas, sendo superior estatisticamente na densidade de 14 plantas. $\mathrm{m}^{-1}$ linear. Da mesma forma, YAMAOKA et al. (1982), avaliando as densidades de 4, 8 e 16 plantas. ${ }^{-}$ ${ }^{1}$ linear, verificaram que a inserção do $1 .^{\circ}$ ramo frutífero é maior conforme o aumento na densidade de plantas na linha.

Quanto ao diâmetro do caule, também não ocorreu interação significativa. Nos espaçamentos de 0,76 e $0,95 \mathrm{~m}$ e na densidade de 5 plantas. $\mathrm{m}^{-1}$ linear, o diâmetro do caule foi significativamente maior. Do mesmo modo, LAMAS e STAUT (1998) verificaram que o diâmetro do caule diminui com o aumento da população de plantas.

O número de ramos vegetativos não foi influenciado pelo espaçamento e densidade ou pela sua interação. O número de plantas na linha, segundo Wright et al. (1998), também não afetou o número de ramos no espaçamento de $0,76 \mathrm{~m}$. Por outro lado, Bednarz et al. (1998) e Lamas e Staut (1998) constataram menor número de ramos vegetativos com o aumento da população de plantas.

Tabela 1. Altura média das plantas na colheita, obtidas em experimento de espaçamento e densidade com o algodoeiro. Ano agrícola 2001/02, Piracicaba (SP)

\begin{tabular}{|c|c|c|c|c|c|}
\hline \multirow{2}{*}{$\begin{array}{l}\text { Espaçamento } \\
\text { entre linhas }\end{array}$} & \multicolumn{5}{|c|}{ Plantas $\mathrm{m}^{-1}$ linear } \\
\hline & 5 & 8 & 11 & 14 & Médias \\
\hline \multicolumn{6}{|c|}{$\overline{\mathrm{m}}$} \\
\hline 0.38 & 85,16 b A & $70,25 \mathrm{~b} \mathrm{~B}$ & 70,33 b B & 81,91 a $A B$ & 76,91 \\
\hline 0,76 & $89,91 \mathrm{ab} A$ & 90,33 a $\mathrm{A}$ & 91,41 a $\mathrm{A}$ & 93,79 a $\mathrm{A}$ & 91,36 \\
\hline 0,95 & 100,75 a $\mathrm{A}$ & 90,87 a $\mathrm{AB}$ & 88,00 a $\mathrm{AB}$ & 82,33 a B & 90,49 \\
\hline Médias & 91,94 & 83,82 & 83,25 & 86,01 & - \\
\hline Espaçamento & - & - & $0,0001^{* *}$ & - & - \\
\hline Densidade & - & - & $0,0344^{*}$ & - & - \\
\hline Espaçamento $x$ Densidade & - & - & $0,0372^{*}$ & - & - \\
\hline CV (\%) & - & - & 8,86 & - & - \\
\hline D.M.S. ${ }^{1}$ & - & - & 13,29 & - & - \\
\hline D.M.S. $^{2}$ & - & - & 14,65 & - & - \\
\hline
\end{tabular}

* **: Significativos aos níveis de $5 \%$ e $1 \%$, respectivamente, pelo teste de $\mathrm{F}$ da análise da variância.

Médias seguidas da mesma letra minúscula, na vertical, não diferem entre si pelo teste de Tukey (5\%).

Médias seguidas da mesma letra maiúscula, na horizontal, não diferem entre si pelo teste de Tukey (5\%).

D.M.S. ${ }^{1}$ : espaçamento dentro de densidade.

D.M.S. ${ }^{2}$ : densidade dentro de espaçamento. 
O número de ramos frutíferos foi influenciado significativamente pelo espaçamento e pela interação entre espaçamento e densidade (Tabela 2). Nos espaçamentos de 0,76 e 0,95 m não houve influência das densidades estudadas, assim como observado por Wright et al. (1998) no espaçamento de $0,76 \mathrm{~m}$. No espaçamento de 0,38 $\mathrm{m}$, na menor densidade de plantas, houve maior número de ramos frutíferos do que na de 11 plantas $\mathrm{m}^{-1}$ linear (Tabela 2). BednARz et al. (1998) e LAMAS e Staut (1998) verificaram que nas maiores populações de plantas houve diminuição no número de ramos frutíferos.
Para o número de internódios, foi verificado efeito do espaçamento e da interação entre espaçamento e densidade (Tabela 3), de modo semelhante ao ocorrido no número de ramos frutíferos (Tabela 2). Os espaçamentos de 0,76 e 0,95 m também não influenciaram significativamente as densidades estudadas. $\mathrm{Na}$ densidade de 11 plantas $\mathrm{m}^{-1}$ linear, o número de internódios no espaçamento de $0,76 \mathrm{~m}$ foi maior que no espaçamento de 0,38 m, concordando com FowLER e RAY (1977) е Jost e CotHREN (2001). Esses resultados discordam do observado por GERIK et al. (1998), os quais não verificaram diferenças no número de internódios entre os espaçamentos ultra-adensado e adensado.

Tabela 2. Número de ramos frutíferos, obtidos em experimento de espaçamentos e densidade com o algodoeiro. Ano agrícola 2001/02, Piracicaba (SP)

\begin{tabular}{|c|c|c|c|c|c|}
\hline \multirow{2}{*}{$\begin{array}{l}\text { Espaçamento } \\
\text { entre linhas }\end{array}$} & \multicolumn{5}{|c|}{ Plantas.m ${ }^{-1}$ linear } \\
\hline & 5 & 8 & 11 & 14 & Médias \\
\hline $\mathrm{m}$ & 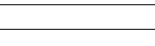 & 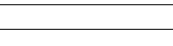 & n. ${ }^{\circ}$ & & \\
\hline 0,38 & 14,04 a $\mathrm{A}$ & 11,54 a $\mathrm{AB}$ & 10,12 b B & 12,04 a $A B$ & 11,94 \\
\hline 0,76 & 13,49 a $\mathrm{A}$ & 13,08 a A & 15,29 a A & 12,37 a $\mathrm{A}$ & 13,56 \\
\hline 0,95 & 13,74 a $\mathrm{A}$ & 13,25 a $\mathrm{A}$ & $11,50 \mathrm{~b} \mathrm{~A}$ & 12,79 a $\mathrm{A}$ & 12,82 \\
\hline Médias & 13,76 & 12,62 & 12,30 & 12,40 & - \\
\hline Espaçamento & - & - & $0,0466^{*}$ & - & - \\
\hline Densidade & - & - & $0,1808^{\text {n.s. }}$ & - & - \\
\hline Espaçamento $x$ Densidade & - & - & $0,0500^{*}$ & - & - \\
\hline CV (\%) & - & - & 13,88 & - & - \\
\hline D.M.S. ${ }^{1}$ & - & - & 3,08 & - & - \\
\hline D.M.S. $^{2}$ & - & - & 3,39 & - & - \\
\hline
\end{tabular}

*, n.s: Significativo e não significativo ao nível de 5\%, respectivamente, pelo teste de $\mathrm{F}$ da análise da variância.

Médias seguidas da mesma letra minúscula, na vertical, não diferem entre si pelo teste de Tukey (5\%).

Médias seguidas da mesma letra maiúscula, na horizontal, não diferem entre si pelo teste de Tukey (5\%).

D.M.S. ${ }^{1}$ : espaçamento dentro de densidade.

D.M.S.' ${ }^{2}$ densidade dentro de espaçamento.

Tabela 3. Número de internódios, obtidos em experimento de espaçamentos e densidade com o algodoeiro. Ano agrícola 2001/ 02. Piracicaba (SP)

\begin{tabular}{|c|c|c|c|c|c|}
\hline \multirow{2}{*}{$\begin{array}{l}\text { Espaçamento } \\
\text { entre linhas }\end{array}$} & \multicolumn{5}{|c|}{ Plantas $\mathrm{m}^{-1}$ linear } \\
\hline & 5 & 8 & 11 & 14 & Médias \\
\hline $\mathrm{m}$ & 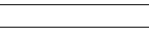 & +2 & $\mathrm{n} .^{\mathrm{o}}$ & & \\
\hline 0,38 & 18,83 a $\mathrm{A}$ & 15,50 a $\mathrm{B}$ & $14,54 \mathrm{~b} \mathrm{~B}$ & 17,29 a $\mathrm{AB}$ & 16,54 \\
\hline 0,76 & 17,37 a $\mathrm{A}$ & 17,71 a $\mathrm{A}$ & 19,54 a $\mathrm{A}$ & 17,29 a $\mathrm{A}$ & 17,98 \\
\hline 0,95 & 18,12 a $\mathrm{A}$ & 17,29 a $A$ & $16,66 \mathrm{~b} \mathrm{~A}$ & 17,87 a $\mathrm{A}$ & 17,49 \\
\hline Médias & 18,11 & 16,83 & 16,91 & 17,48 & - \\
\hline Espaçamento & - & - & $0,0355^{*}$ & - & - \\
\hline Densidade & - & - & $0,1624^{\text {n.s. }}$ & - & - \\
\hline Espaçamento $x$ Densidade & - & - & $0,0078^{* *}$ & - & - \\
\hline $\mathrm{CV}(\%)$ & - & - & 8,76 & - & - \\
\hline D.M.S. ${ }^{1}$ & - & - & 2,64 & - & - \\
\hline D.M.S. $^{2}$ & - & - & 2,91 & - & - \\
\hline
\end{tabular}

*,**, n.s.: Significativos aos níveis de 5\% e 1\% e não significativo, respectivamente, pelo teste de $\mathrm{F}$ da análise da variância. Médias seguidas da mesma letra minúscula, na vertical, não diferem entre si pelo teste de Tukey (5\%).

Médias seguidas da mesma letra maiúscula, na horizontal, não diferem entre si pelo teste de Tukey (5\%).

D.M.S. ${ }^{1}$ : espaçamento dentro de densidade.

D.M.S. ${ }^{2}$ : densidade dentro de espaçamento. 


\section{CONCLUSÕES}

1. Quanto menor o espaçamento entrelinhas e maior a densidade de plantas na linha, maior será o índice de área foliar e menor o diâmetro do caule devido ao estiolamento das plantas.

2. Quanto maior o espaçamento e menor a densidade, maior a massa seca da parte aérea e a altura média das plantas.

3. Maior densidade de plantas na linha condiciona maior altura de inserção do $1 .^{\circ}$ ramo frutífero.

4. Independentemente do espaçamento e da densidade, o número de ramos vegetativos não é alterado, enquanto o número de ramos frutíferos e o número de internódios diminuem com o aumento da população de plantas, o que é compensado pelo aumento do número desses ramos por unidade de área.

5. Diferentes configurações de semeadura alteram o crescimento e o desenvolvimento das plantas de algodoeiro e, conseqüentemente, o manejo da cultura.

\section{AGRADECIMENTOS}

À Coordenação de Aperfeiçoamento de Pessoal de Nível Superior (CAPES), pela concessão da bolsa de mestrado, à primeira autora. À Escola Superior de Agricultura "Luiz de Queiroz"/ Universidade de São Paulo, pelo apoio e pela infraestrutura. Ao Engenheiro Agrônomo Dr. Edson Roberto Teramoto, responsável pela área experimental agrícola do Departamento de Produção Vegetal/Setor de Agricultura da USP/ESALQ.

\section{REFERÊNCIAS}

BEDNARZ, C.W.; BAKER, S.H.; BROWN, S.M.; BRIDGES, D. Effects of plant population on growth and development of cotton in south-Georgia. In: BELTWIDE COTTON CONFERENCE, 1998, San Diego. Proceedings... Memphis: National Cotton Council of America, 1998. v.2, p.1450.

FOWLER, J.L.; RAY, L.L. Response of two cotton genotypes to five equidistant spacing patterns. Agronomy Journal, Madison, v.69, n.5, p.733-738, 1977.

GERIK, T.J.; LEMON, R.G.; FAVER, K.L.; HOELEWYN, T.A.; JUNGMAN, M. Performance of ultra-narrow row cotton in central Texas. In: BELTWIDE COTTON CONFERENCE, 1998, San Diego. Proceedings... Memphis: National Cotton Council of America, 1998. v.2, p.1406-1409.
GRIDI-PAPP, I.L.; CIA, E.; FUZATTO, M.G.; SILVA, N.M. da; FERRAZ, C.A.M.; CARVALHO, N. de; CARVALHO, L.H.; SABINO, N.P.; KONDO, J.J.; PASSOS, S.M.G.; CHIAVEGATO, G.I.; CAMARGO, P.P. de; CAVALERI, P.A. Manual do produtor de algodão. São Paulo: Bolsa de Mercadorias \& Futuros, 1992. 158 p.

JOST, P.H.; COTHREN, J.T. Ultra-narrow row and conventionally spaced cotton: growth and yield comparisons. In: BELTWIDE COTTON CONFERENCE, 1999b, Orlando. Proceedings... Memphis: National Cotton Council of America, 1999. v.1, p.559.

JOST, P.H.; COTHREN, J.T. Phenotypic alterations and crop maturity differences in ultra-narrow row and conventionally spaced cotton. Crop Science, Madison, v.41, n.4, p.1150-1159, 2001.

LAMAS, F.M.; STAUT, L.A. Espaçamento e densidade. In: EMBRAPA/CNPA. Algodão: informações técnicas. Dourados: EMBRAPA, 1998. p.103-105. (Circular técnica, 7)

PASSOS, S.M.G. Algodão. Campinas: Instituto Campineiro de Ensino Agrícola, 1977. 424p.

REEVES, W. Sistemas de preparo conservacionistas para algodão. In: ENCONTRO NACIONAL DE PLANTIO DIRETO NA PALHA, 7., Foz do Iguaçu, 2000. Resumos... Foz do Iguaçu: Federação Brasileira de Plantio Direto na Palha, 2000. p.90-93.

RIGHI, N.R.; FERRAZ, C.A.M.; CORRÊA, D.M. VII Cultura. In: INSTITUTO BRASILEIRO DE POTASSA. Cultura e adubação do algodoeiro. São Paulo, 1965. p.255-317.

VIANELLO, R.L.; ALVES, A.R. Meteorologia básica e aplicações. Viçosa: Impressão Universitária, 1991. p.395-399.

WEIR, B.L. Narrow row cotton distribution and rationale. In: BELTWIDE COTTON CONFERENCE, 1996, Nashville. Proceedings... Memphis: National Cotton Council of America, 1996. v.1, p.65-66.

WRIGHT, S.D.; VARGAS, R.; WEIR, B.; MUNK, D.; ROBERTS, B.; HUTMACHER, B.; MARTIN-DUVALL, T.M.; KEELEY, M.; BANUELOS, G.; JIMENEZ, M.R. Effect of planting date and density on San Joaquim Valley cotton. In: BELTWIDE COTTON CONFERENCE, 1998, San Diego. Proceedings... Memphis: National Cotton Council of America, 1998. v.2, p.1451.

YAMAOKA, R.S.; PIRES, J.R.; ALMEIDA, W.P. de. Efeito da densidade de plantas de algodoeiro sobre a inserção de ramos frutíferos. In: REUNIÃO NACIONAL DO ALGODÃO, 2., Salvador, 1982. Resumos.... Campina Grande: EMBRAPA, CNPA, 1982. p.109. 